\title{
Comparative study of direct and inverse problems of cracked beams
}

\author{
CHETTAH Mahieddine ${ }^{1}$ and LASSOUED Rachid ${ }^{1}$ \\ ${ }^{1}$ University of brothers Mentouri-Constantine 1, Department of Civil Engineering, \\ Laboratory of Materials and Durability of Constructions, Constantine, Algeria
}

\begin{abstract}
In recent decades, the analysis and evaluation of the cracked structures were hot spots in several engineering fields and has been the subject of great interest with important and comprehensive surveys covering various methodologies and applications, in order to obtain reliable and effective methods to maintain the safety and performance of structures on a proactive basis. The presence of a crack, not only causes a local variation in the structural parameters (e.g., the stiffness of a beam) at its location, but it also has a global effect which affects the overall dynamic behavior of the structure (such as the natural frequencies). For this reason, the dynamic characterization of the cracked structures can be used to detect damage from non-destructive testing. The objective of this paper is to compare the accuracy and ability of two methods to correctly predict the results for both direct problem to find natural frequencies and inverse problem to find crack's locations and depths of a cracked simply supported beam. Several cases of crack depths and crack locations are investigated. The crack is supposed to remain open. The Euler-Bernoulli beam theory is employed to model the cracked beam and the crack is represented as a rotational spring with a sectional flexibility. In the first method, the transfer matrix method is used; the cracked beam is modeled as two uniform sub-segments connected by a rotational spring located at the cracked section. In the second method which is based on the Rayleigh's method, the mode shape of the cracked beam is constructed by adding a cubic polynomial function to that of the undamaged beam. By applying the compatibility conditions at crack's location and the corresponding boundary conditions, the general forms of characteristic equations for this cracked system are obtained. The two methods are then utilized to determine the locations and depths by using any two natural frequencies of a cracked simply supported beam obtained from measurements as inputs. The two approaches are compared with a number of numerical examples for simply supported beams including one crack. The theoretical results show that the accuracy of the Rayleigh's method to predict natural frequencies decreases for higher modes when crack depth increases. It is also found that for the inverse problem, the transfer matrix method show a good agreement with those obtained from previous works done in this field.
\end{abstract}

\section{Introduction}

In the areas of civil, mechanical and aerospace engineering, the structure must fulfill some requirements such as durability, resistance, working safely and the capacity to satisfy the needs of users during its operational lifetime, which requires a continuous monitoring to detect possible damages (e.g., cracks) that allows an assessment of its state from the current structural conditions also provide a certain forecast of its future performance and information for maintenance, in order to verify its integrity and compliance with applicable regulations. The dynamic behavior of cracked beams has been studied by various analytical, numerical and experimental methods. Dimarogonas [1] and Gasch [2] presented comprehensive reviews of the dynamics of cracked structures. When a structural component is subjected to cracks, it lead to changes in structural parameters (e.g., the stiffness of a structural member such as beam elements), which, in turn, change dynamic behavior (such as natural frequencies and mode shapes).

Damage detection of beam elements has two different aspects: the first is to study the effects of cracks on the structure parameters (mass, damping and stiffness), from a detailed numerical model based on the geometric and material properties of the structure as a direct problem. Different approaches were used to model cracks in beams, such as equivalent reduced cross- section, local bending moment and local flexibility. Ostachowicz and Krawczuk [3] gave comprehensive surveys of crack modeling approaches. Friswell and Penny [4] compare the different approaches to crack modeling, and demonstrate that for structural health monitoring using low frequency vibration, simple models of crack flexibility based on beam elements are adequate. A common approach is representing the crack by a rotational spring, but with different formulas to modeling the effect of a crack on the local flexibility [5-7]. 
The second can be regarded as how to detect the location and depth of the cracks, from experimentally measured data, by analyzing the changes in the vibration characteristics of the structure (natural frequencies, mode shapes, etc.), as an inverse problem. In the field of inverse problems, both analytical and numerical classes of crack detection problem were usually based on changes of natural frequencies, measuring dynamic flexibility or comparison of mode shapes [8-10]. Among the aforementioned crack identification methods, greater attention has been devoted to solve the crack problem based on changes of fundamental frequencies. Liang et al [11] developed a frequency contour plot method based on measurements of the first three natural frequencies to detect a crack in a beam. Nandwana and Maiti [12] have been extended the method to stepped beams. Lee [13] used a combination of the finite element method and the Newton-Raphson procedure to identify multiple cracks. Attar [14] used a transfer matrix method to solve a similar problem with general form of boundary conditions.

The objective of this paper is to compare the accuracy and ability of two methods to correctly predict the results for both direct and inverse problem of a simply supported beam with one crack. Several cases of crack depths and crack locations are studied. The crack is supposed to remain open. The Euler-Bernoulli beam theory is employed to model the cracked beam and the crack is represented as a rotational spring. In the first method, the transfer matrix method (TMM) is used; the cracked beam is modeled as two uniform sub-segments connected by a rotational spring located at the cracked section. The second one, based on the Rayleigh's method (RM), the mode shapes of the cracked beam is constructed by adding a cubic polynomial function to that of the undamaged beam. By applying the compatibility conditions at crack's location and the corresponding boundary conditions, the general forms of characteristic equations for this cracked beam system are obtained. The two methods are then utilized to determine the locations and depths by using any two natural frequencies of a cracked simply supported beam obtained from measurements as input. The two approaches are compared with a number of numerical examples for simply supported beams including one crack. The results show a good agreement with those obtained from previous works done in this fields.

\section{Theoretical model}

A simply supported cracked beam as shown in figure 1 has length $L$ and longitudinal uniform rectangular cross section with height of $h$, width of $b$ and one open traversed crack of depth $a$ located at position $X_{1}=L_{1}$ from the left support of the beam. The beam is modeled as an Euler-Bernoulli in two segments joined by a rotational springs. The transverse deflection function of the beam at axial coordinate $X$ and time $T$, is denoted by $W_{i}(X, T)$ for two intervals of $i=1$ for $0<X<X_{1}$ and $i=2$ for $X_{1}<X<L$.
The differential equation of motion for each segment is:

$E I \frac{\partial^{4} W_{i}(X, T)}{\partial X^{4}}+\rho A \frac{\partial^{2} W_{i}(X, T)}{\partial T^{2}}=0 ; i=1,2$

$E ; \rho ; A=b h$ and $I=b h^{3} / 12$ are Young's modulus, density of the material, cross sectional area of the rectangular beam and moment of inertia of the uniform and homogeneous beam, respectively.

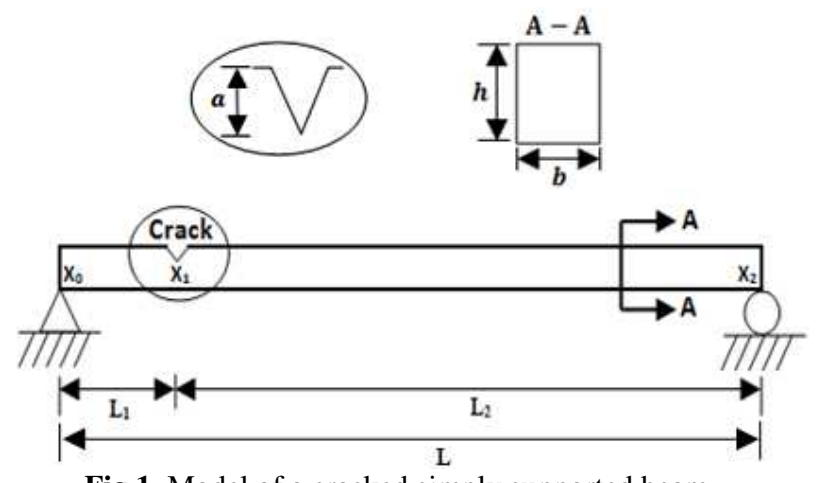

Fig.1. Model of a cracked simply supported beam.

From the above equations, the following quantities are introduced for non-dimensional analysis:

$w_{i}=\frac{W_{i}}{L} \quad ; \quad t=T ; x_{i}=\frac{X_{i}}{L} \quad ; \quad l_{i}=\frac{L_{i}}{L}$

Eq. (1) can be changed to:

$\frac{E I}{L^{4}} \frac{\partial^{4} w_{i}(x, t)}{\partial x^{4}}+\rho A \frac{\partial^{2} w_{i}(x, t)}{\partial t^{2}}=0 ; i=1,2$

The boundary conditions of this simply supported beam as non-dimensional form are:

$x=O\left\{\begin{array}{l}\varphi_{1}(0)=0 \\ M_{1}(0)=0\end{array} ; \quad x=1\left\{\begin{array}{l}\varphi_{2}(1)=0 \\ M_{2}(1)=0\end{array}\right.\right.$

The continuous conditions at the crack position as nondimensional form are:

$x=l_{1}\left\{\begin{array}{c}\varphi_{1}\left(l_{1}\right)=\varphi_{2}\left(l_{1}\right) \\ \theta_{1}\left(l_{1}\right)=\theta_{2}\left(l_{1}\right)-\eta M_{2}\left(l_{1}\right) \\ M_{1}\left(l_{1}\right)=M_{2}\left(l_{1}\right) \\ T_{1}\left(l_{1}\right)=T_{2}\left(l_{1}\right)\end{array}\right.$

$\varphi, \theta, M$ and $T$ are respectively the displacement, slope, bending moment and shear force expressions of the cracked beam.

Where $\eta=\frac{E I c}{L}$, is the non-dimensional cracked beam sectional flexibility, which is related to the ratio of crack depth to the beam height, $\xi=a / h$. It can be expressed as [7]:

$$
\begin{aligned}
& \eta=\frac{h}{L} 6 \pi\left(1-v^{2}\right) f(\xi) \\
& f(\xi)=0.6272 \xi^{2}-1.04533 \xi^{3}+4.5948 \xi^{4}- \\
& 9.9736 \xi^{5}+20.2948 \xi^{6}-33.0351 \xi^{7}+47.1063 \xi^{8}- \\
& 40.7556 \xi^{9}+19.6 \xi^{10}
\end{aligned}
$$

Where $v$ is the Poisson's ratio. 


\section{Direct problem}

\subsection{Transfer matrix method}

In order to determine natural frequencies and mode shapes, the free vibration solution can be found using the method of separation of variables as:

$w_{i}(x, t)=\varphi_{i}(x) e^{j \omega t} \quad i=1,2$

Using Eq. (7) and applied to Eq. (3) yields to:

$\varphi_{i}^{(4)}(x)-\beta^{4} \varphi_{i}(x)=0 \quad i=1,2$

Where:

$\beta^{4}=\frac{L^{4} \rho A \omega^{2}}{E I}$

$\omega$ is the natural frequency of the entire uniform beam. The general solution of the ordinary differential equation (9), for each segment, can be expressed as:

$\varphi_{i}(x)=A_{i} \sin \left[\beta\left(x-x_{i-1}\right)\right]+B_{i} \cos \left[\beta\left(x-x_{i-1}\right)\right]+$

$C_{i} \sinh \left[\beta\left(x-x_{i-1}\right)\right]+D_{i} \cosh \left[\beta\left(x-x_{i-1}\right)\right]$

In which, $A_{i}, B_{i}, C_{i}$, and $D_{i}$ are unknown constants associated with the $i$ th segment $(i=1,2)$.

By substituting the boundary and continuous equations (4) and (5) into Eq. (10) for $(i=1,2)$ respectively and calculating the determinant of the matrix, the characteristic equation in this case assumes the form:

$\eta \beta_{n}\left[\sinh \left(\beta_{n} l_{1}\right) \sinh \left(\beta_{n} l_{2}\right) \sin \left[\beta_{n}\left(l_{1}+l_{2}\right)\right]-\right.$ $\left.\sin \left(\beta_{n} l_{1}\right) \sin \left(\beta_{n} l_{2}\right) \sinh \left[\beta_{n}\left(l_{1}+l_{2}\right)\right]\right]+2 \sinh \left[\beta_{n}\left(l_{1}+\right.\right.$ $\left.\left.l_{2}\right)\right] \sin \left[\beta_{n}\left(l_{1}+l_{2}\right)\right]=0$

The above characteristic equation is solved with the help of MATLAB software using vpasolve function. Ones the parameters $\beta_{n}$ are obtained, the natural frequencies of the cracked beam can be obtained using the following equation:

$\omega_{c n}=\left(\frac{\beta_{n}}{L}\right)^{2} \sqrt{\frac{E I}{\rho A}}$

Where, $\mathrm{n}$ is the mode range.

The constants $A_{i}, B_{i}, C_{i}$, and $D_{i}$ can also be founded directly by the substitution method and normalizing it for the constant $A_{1}$.

\subsection{The Rayleigh's method}

In this section, by extending the Rayleigh's method as used by Fernandez-Saez et al. [15], the fundamental frequencies and mode shapes of a simply supported beam with one crack can be obtained. The transverse deflection of the cracked beam is constructed by adding a cubic polynomial function to that of the un-cracked beam, to represent crack effects on displacement of the section of the cracked beam located to the left and the right of the crack respectively.
The general expression for the mode shapes of cracked beam is:

$$
\begin{gathered}
\varphi_{i}(X)=\sin \left(\frac{n \pi X}{L}\right)+A_{i} X^{3}+B_{i} X^{2}+C_{i}+D_{i} \\
0 \leq X \leq L_{i} \\
\varphi_{i+1}(X)=\sin \left(\frac{n \pi X}{L}\right)+A_{i+1} X^{3}+B_{i+1} X^{2}+C_{i+1} \\
+D_{i+1} \quad L_{i} \leq X \leq L
\end{gathered}
$$

By applying the boundary conditions of this simply supported beam and the continuous conditions at the point of crack, the general expression for the mode shapes of cracked beam which is presented in nondimensional form, can be obtained as:

$$
\begin{gathered}
\varphi_{i}(x)=\sin (n \pi x)+\eta\left[(n \pi)^{2} \sin \left(n \pi l_{i}\right)\right]\left[1-l_{i}\right] x \\
0 \leq x \leq l_{i} \\
\varphi_{i+1}(x)=\sin (n \pi x)+\eta\left[(n \pi)^{2} \sin \left(n \pi l_{i}\right)\right] l_{i}[1-x] \\
l_{i} \leq x \leq 1
\end{gathered}
$$

The Rayleigh's method is used, so by equating the maximum values of potential energy $U_{\max }$ and kinetic energy $T_{\max }$ of the cracked simply supported beam, the natural frequencies can be obtained.

The maximum potential energy can be calculated as:

$U_{\max }=\frac{E I}{2}\left[\int_{0}^{L_{i}}\left(\frac{M_{i}}{E I}\right)^{2} d X+\int_{L_{i}}^{L}\left(\frac{M_{i+1}}{E I}\right)^{2} d X\right]+$
$\left[\frac{1}{2}\left(\theta_{i+1}-\theta_{i}\right) M\right]_{X=L_{i}}=\frac{E I(n \pi)^{4}}{4 L^{3}}\left[1+\eta \gamma_{n}\right]$

The maximum kinetic energy can be calculated as:

$T_{\max }=\frac{\rho A \omega_{c n}^{2}}{2}\left[\int_{0}^{L_{i}}\left(\varphi_{i}\right)^{2} d X+\int_{L_{i}}^{L}\left(\varphi_{i+1}\right)^{2} d X\right]=$
$\frac{\rho A \omega_{c n}^{2}}{2} \frac{L}{2}\left[1+\eta \gamma_{n}\left[2+\eta\left(\frac{(n \pi)^{4}}{3}\right) l_{i}^{2}\left(1-l_{i}\right)^{2}\right]\right]$

By equating equations (15) and (16), the nth-natural angular frequency of cracked simply supported beam is:

$\omega_{c n}=\omega_{n}\left[\frac{1+\eta \gamma_{n}}{1+\eta \gamma_{n}\left[2+\eta\left(\frac{(n \pi)^{4}}{3}\right) l_{i}^{2}\left(1-l_{i}\right)^{2}\right]}\right]^{1 / 2}$

Where:

$\left\{\begin{array}{l}\omega_{n}=\left(\frac{n \pi}{L}\right)^{2} \sqrt{\frac{E I}{\rho A}} \\ \gamma_{n}=2 \sin ^{2}\left(n \pi l_{i}\right)\end{array}\right.$

\section{Inverse problem}

The inverse problem consists of determining the damage parameters (the location and the extent of a crack) when a certain number of measured natural frequencies are known.

The procedures for crack identification for the two methods are given below: 


\subsection{Transfer matrix method}

To obtain the unknown parameters $\eta$ and $l_{1}$, the following steps are used, which are similar to the method used in [16].

- The Eq. (11) is rewritten as:

$\eta \beta_{n}\left[\sinh \left(\beta_{n} l_{1}\right) \sinh \left(\beta_{n} l_{2}\right) \sin \left[\beta_{n}\left(l_{1}+l_{2}\right)\right]-\right.$ $\left.\sin \left(\beta_{n} l_{1}\right) \sin \left(\beta_{n} l_{2}\right) \sinh \left[\beta_{n}\left(l_{1}+l_{2}\right)\right]\right]=$

$-2 \sinh \left[\beta_{n}\left(l_{1}+l_{2}\right)\right] \sin \left[\beta_{n}\left(l_{1}+l_{2}\right)\right]$

- Calculating $\beta_{1}$ and $\beta_{2}$ from Eq. (9) by utilizing the two measured frequencies $\omega_{c 1}$ and $\omega_{c 2}$;

- Substituting $\beta_{1}$ and $\beta_{2}$ separately into Eq. (19) and dividing these two equations, the non-dimensional crack section flexibility $\eta$ can then be cancelled out and the following identification equation can be obtained:

$\frac{\beta_{1}\left[\sinh \left(\beta_{1} l_{1}\right) \sinh \left(\beta_{1} l_{2}\right) \sin \left(\beta_{1}\right)-\sin \left(\beta_{1} l_{1}\right) \sin \left(\beta_{1} l_{2}\right) \sinh \left(\beta_{1}\right)\right]}{\beta_{2}\left[\sinh \left(\beta_{2} l_{1}\right) \sinh \left(\beta_{2} l_{2}\right) \sin \left(\beta_{2}\right)-\sin \left(\beta_{2} l_{1}\right) \sin \left(\beta_{2} l_{2}\right) \sinh \left(\beta_{2}\right)\right]}=$ $\sinh \left(\beta_{1}\right) \sin \left(\beta_{1}\right)$

$\overline{\sinh \left(\beta_{2}\right) \sin \left(\beta_{2}\right)}$

- The position of the crack $l_{1}$, can be determinate with the help of MATLAB software using solve function;

- After obtaining $l_{1}$, the non-dimensional crack sectional flexibility $\eta$ can be determined from Eq. (11), and the crack depth $a$ can then be obtained from Eq. (6).

\subsection{The Rayleigh's method}

In this section, the inverse problem can be solved by using Eq. (14) in Ref. [17], as:

$\frac{\Delta \omega_{n}}{\omega_{n}}=\frac{\eta \gamma_{n}}{2}$

Where:

$\Delta \omega_{n}=\omega_{n}-\omega_{c n}$

- Calculating $\omega_{1}$ and $\omega_{2}$ from Eq. (18) and by utilizing the two measured frequencies $\omega_{c 1}$ and $\omega_{c 2}, \Delta \omega_{1}$ and $\Delta \omega_{2}$ can be obtained;

- Substituting $\frac{\Delta \omega_{1}}{\omega_{1}}$ and $\frac{\Delta \omega_{2}}{\omega_{2}}$ separately into Eq. (21) and dividing these two equations, the non-dimensional crack section flexibility $\eta$ can then be cancelled out and the following identification equation can be obtained:

$\frac{\Delta \omega_{1}}{\omega_{1}} \frac{\omega_{2}}{\Delta \omega_{2}}=\frac{\gamma_{1}}{\gamma_{2}}$

- The position of the crack $l_{1}$, can be determinate with the help of MATLAB software using solve function;

- After obtaining $l_{1}$, the non-dimensional crack sectional flexibility $\eta$ can be determined from Eq. (11) and the crack depth $a$ can then be obtained from Eq. (6).

\section{Results and discussion}

In order to validate the study presented in this paper and to show the effectiveness and also the limitations of the two methods, results obtained by applying these two methods are compared with the available data for single open cracked simply supported beam.

The material parameters for an undamaged simply supported beam are: $E=2.1 \times 10^{11} \mathrm{~Pa}, \quad v=0.3$, $\rho=7860 \mathrm{~kg} / \mathrm{m}^{3}$, and its dimensions are: $L=0.5 \mathrm{~m}$, $h=0.02 \mathrm{~m}, b=0.012 \mathrm{~m}$.

Table 1 shows comparison of the first three natural frequencies of undamaged beam obtained from the two methods with what computed by [18]. The results show that both methods yield the same exact frequencies and agree well with those obtained from [18].

Table 1. Comparison of frequencies of undamaged beam, obtained by two methods and results given by Ref. [18].

\begin{tabular}{|c|c|c|c|}
\hline & $\omega_{1}$ & $\omega_{2}$ & $\omega_{3}$ \\
\hline$[18]$ & 1178.160 & 4712.577 & 10603.252 \\
\hline 1st method & 1178.141 & 4712.566 & 10603.274 \\
\hline 2nd method & 1178.141 & 4712.566 & 10603.274 \\
\hline
\end{tabular}

\subsection{Direct problem}

The lowest three natural frequencies of transverse vibration are calculated by the two methods, and compared with what reported by [19] for the damaged beam with one crack. The results for various crack locations and depths, given in Table 2, show a reasonable agreement between the two methods results and those achieved by [19] for all cases of the cracked beam, but the difference between natural frequencies increases for higher order modes.

Table 2. Comparison of first three natural frequencies of cracked beams, obtained by two methods and results given by Ref. [19] for various crack location and depth.

\begin{tabular}{|c|c|c|c|c|c|c|}
\hline \multirow{2}{*}{$\begin{array}{c}\text { Case } \\
\text { number }\end{array}$} & \multirow{2}{*}{$l_{1}$} & \multirow{2}{*}{$\xi$} & \multirow{2}{*}{ Methods } & \multicolumn{3}{|c|}{ Natural frequencies, $\omega_{c n}(\mathrm{rad} / \mathrm{s})$} \\
\hline & & & & $\omega_{c 1}$ & $\omega_{c 2}$ & $\omega_{c 3}$ \\
\hline \multirow{3}{*}{1} & \multirow{3}{*}{0.1} & \multirow{3}{*}{0.1} & [19] & 1177.660 & 4705.604 & 10573.663 \\
\hline & & & TMM & 1177.709 & 4706.310 & 10576.662 \\
\hline & & & $\mathrm{RM}$ & 1177.709 & 4706.234 & 10574.665 \\
\hline \multirow{3}{*}{2} & \multirow{3}{*}{0.2} & \multirow{3}{*}{0.3} & [19] & 1162.672 & 4557.815 & 10281.642 \\
\hline & & & TMM & 1164.032 & 4570.851 & 10306.810 \\
\hline & & & $\mathrm{RM}$ & 1164.036 & 4518.740 & 9675.567 \\
\hline \multirow{3}{*}{3} & \multirow{3}{*}{0.3} & \multirow{3}{*}{0.2} & [19] & 1165.559 & 4644.925 & 10587.306 \\
\hline & & & TMM & 1166.720 & 4651.012 & 10588.734 \\
\hline & & & $\mathrm{RM}$ & 1166.721 & 4631.081 & 10562.019 \\
\hline \multirow{3}{*}{4} & \multirow{3}{*}{0.3} & \multirow{3}{*}{0.3} & {$[19]$} & 1149.363 & 4563.174 & 10568.267 \\
\hline & & & TMM & 1151.851 & 4575.368 & 10571.089 \\
\hline & & & $\mathrm{RM}$ & 1151.858 & 4477.435 & 10428.520 \\
\hline \multirow{3}{*}{5} & \multirow{3}{*}{0.4} & \multirow{3}{*}{0.2} & [19] & 1160.876 & 4686.538 & 10544.768 \\
\hline & & & TMM & 1162.459 & 4688.896 & 10550.062 \\
\hline & & & $\mathrm{RM}$ & 1162.460 & 4677.927 & 10429.644 \\
\hline \multirow{3}{*}{6} & \multirow{3}{*}{0.4} & \multirow{3}{*}{0.4} & [19] & 1105.78 & 4608.053 & 10369.596 \\
\hline & & & TMM & 1110.837 & 4614.974 & 10384.937 \\
\hline & & & $\mathrm{RM}$ & 1110.847 & 4416.368 & 8607.153 \\
\hline \multirow{3}{*}{7} & \multirow{3}{*}{0.5} & \multirow{3}{*}{0.2} & [19] & 1159.108 & 4712.566 & 10436.419 \\
\hline & & & TMM & 1160.849 & 4712.566 & 10451.318 \\
\hline & & & $\mathrm{RM}$ & 1160.849 & 4712.566 & 10105.454 \\
\hline \multirow{3}{*}{8} & \multirow{3}{*}{0.5} & \multirow{3}{*}{0.4} & [19] & 1099.580 & 4712.566 & 9964.053 \\
\hline & & & TMM & 1104.527 & 4712.566 & 10003.928 \\
\hline & & & $\mathrm{RM}$ & 1104.528 & 4712.566 & 6723.939 \\
\hline
\end{tabular}


Figure 2 represents plots of the corresponding mode shapes, using the two methods.

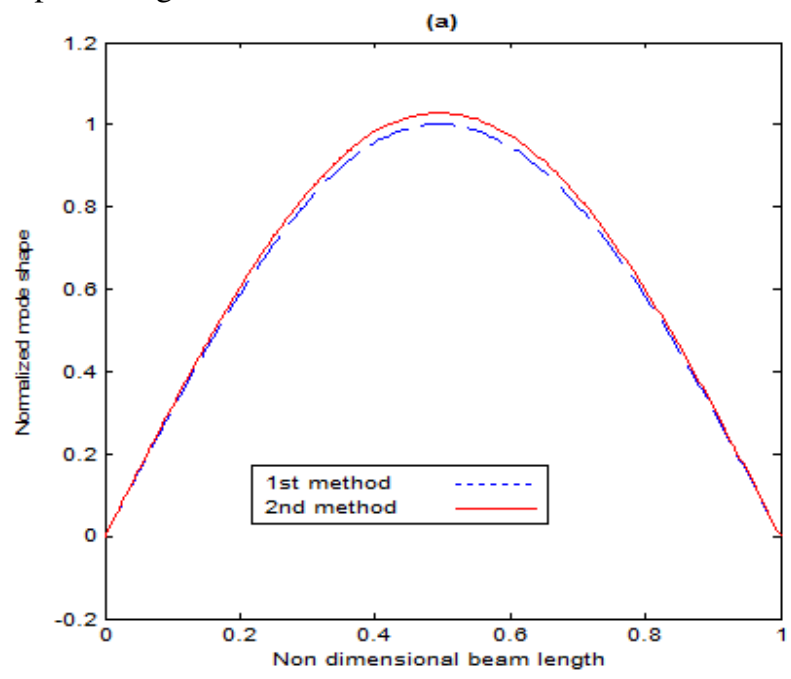

(b)

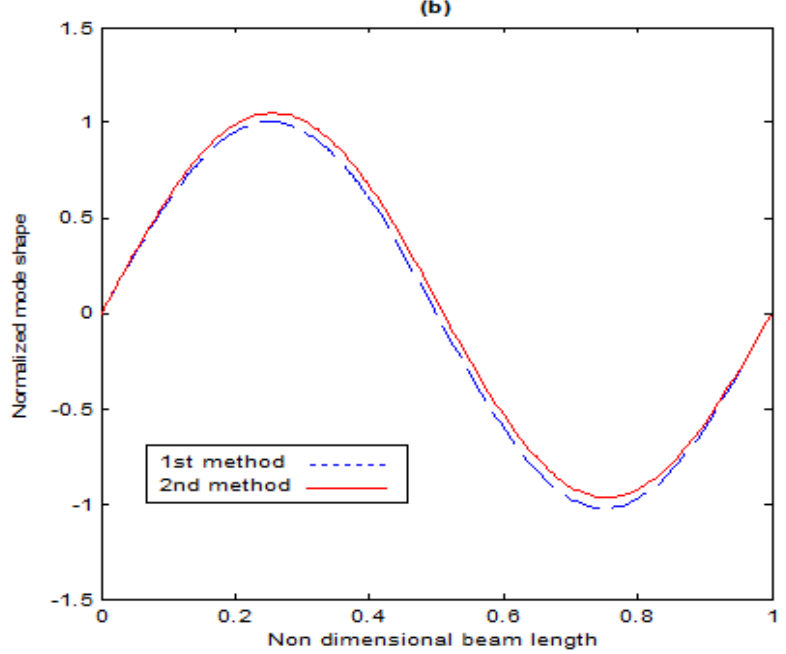

(c)

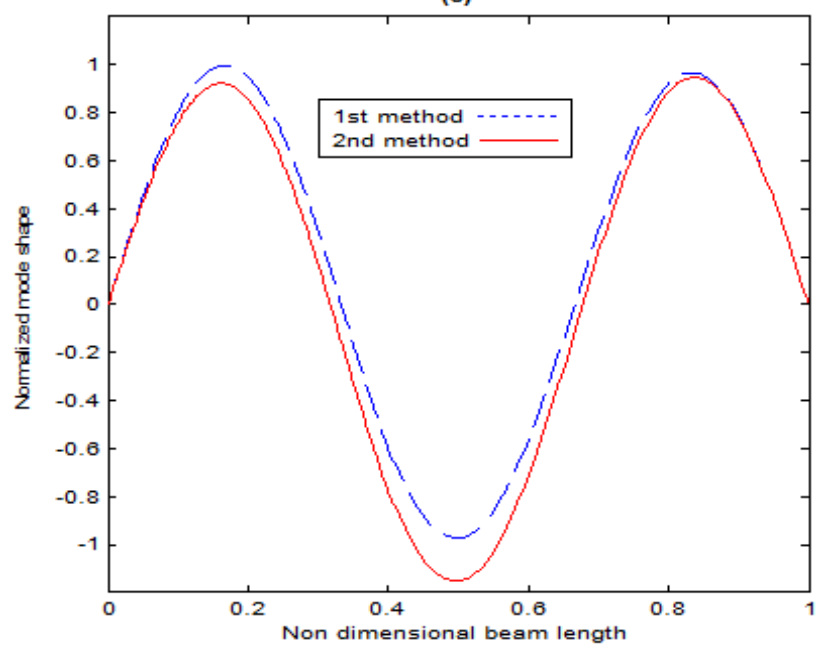

Fig.2. Comparison of the two methods for first three mode shapes of beam having one crack. $l_{1}=0.4 ; \xi=0.2$. (a) $1^{\text {st }}$ mode shape, (b) $2^{\text {nd }}$ mode shape and (c) $3^{\text {rd }}$ mode shape.

Table 3 shows comparison of the first three relative errors of natural frequencies (R.E.N.F) obtained by the two methods for various crack location and depth, where:

R.E. N.F $(\%)=\left|\frac{\omega_{c n 1}-\omega_{c n 2}}{\omega_{c n 1}}\right| \times 100$ $\omega_{c n 1}$ and $\omega_{c n 2}$ are the nth calculated natural frequencies by the first and the second methods respectively.

As can be seen, the two methods can approximate first natural frequencies of cracked beam with high accuracy for various crack locations and depths. Also the two methods can approximate second natural frequencies of cracked beam with maximum error less than $5 \%$ in the 6th case when the crack location/ depth are respectively $(0.4,0.4)$, but the difference between natural frequencies increases for the third mode of vibration.

Table 3. Comparison of relative error between first three natural frequencies, obtained by the two methods for various crack location and depth.

\begin{tabular}{|c|c|c|c|}
\hline $\begin{array}{c}\text { Case } \\
\text { number }\end{array}$ & $\begin{array}{c}1 \text { st R.E.N.F } \\
(\%)\end{array}$ & $\begin{array}{c}\text { 2nd R.E.N.F } \\
(\%)\end{array}$ & $\begin{array}{c}\text { 3rd R.E.N.F } \\
(\%)\end{array}$ \\
\hline 1 & $\approx 0$ & 0.001 & 0.018 \\
\hline 2 & $\approx 0$ & 1.140 & 6.124 \\
\hline 3 & $\approx 0$ & 0.428 & 0.252 \\
\hline 4 & $\approx 0$ & 2.140 & 1.348 \\
\hline 5 & $\approx 0$ & 0.233 & 1.141 \\
\hline 6 & $\approx 0$ & 4.303 & 17.118 \\
\hline 7 & $\approx 0$ & $\approx 0$ & 3.309 \\
\hline 8 & $\approx 0$ & $\approx 0$ & 32.787 \\
\hline
\end{tabular}

\subsection{Inverse problem}

The inverse problem is solved by using the exact first three frequencies used in [19] as inputs from various cases.

Table 4.a. Comparison of predicted and actual crack parameters for various crack location and depth by using $\omega_{c 1}-\omega_{c 2}$.

\begin{tabular}{|c|c|c|c|c|c|}
\hline \multirow{2}{*}{ Case } & \multirow{2}{*}{ Methods } & \multicolumn{4}{|c|}{$\omega_{c 1}-\omega_{c 2}$} \\
\cline { 3 - 6 } & & $l_{1}^{*}$ & Error $(\%)$ & $\xi^{*}$ & Error $(\%)$ \\
\hline \multirow{2}{*}{1} & Method1 & 0.1002 & 0.2473 & 0.1054 & 5.4543 \\
\cline { 3 - 6 } & Method2 & 0.1005 & 0.5028 & 0.1500 & 50.0562 \\
\hline \multirow{2}{*}{2} & Method1 & 0.2000 & 0.0041 & 0.3128 & 4.2935 \\
\cline { 2 - 6 } & Method2 & 0.2097 & 4.8554 & 0.4007 & 33.5849 \\
\hline \multirow{2}{*}{3} & Method1 & 0.2999 & 0.0004 & 0.2099 & 4.9612 \\
\cline { 2 - 6 } & Method2 & 0.3031 & 1.0639 & 0.2871 & 43.5758 \\
\hline \multirow{2}{*}{4} & Method1 & 0.2999 & 0.0005 & 0.3128 & 4.2947 \\
\cline { 2 - 6 } & Method2 & 0.3070 & 2.3661 & 0.4056 & 35.2128 \\
\hline \multirow{2}{*}{5} & Method1 & 0.4000 & 0.0002 & 0.2099 & 4.9622 \\
\cline { 2 - 6 } & Method2 & 0.4006 & 0.1726 & 0.2880 & 44.0040 \\
\hline \multirow{2}{*}{6} & Method1 & 0.3999 & 0.0001 & 0.4126 & 3.1733 \\
\cline { 2 - 6 } & Method2 & 0.4028 & 0.7158 & 0.5087 & 27.1855 \\
\hline \multirow{2}{*}{7} & Method1 & 0.5003 & 0.0652 & 0.2099 & 4.9610 \\
\cline { 2 - 6 } & Method2 & 0.5 & 0 & 0.2878 & 43.9407 \\
\hline \multirow{2}{*}{8} & Method1 & 0.4998 & 0.0322 & 0.4115 & 2.8772 \\
\cline { 2 - 6 } & Method2 & 0.5 & 0 & 0.5073 & 26.8415 \\
\hline
\end{tabular}

Tables $4 \mathrm{a}-4 \mathrm{c}$ show the comparison of predicted $\left(l_{1}^{*}, \xi^{*}\right)$ and actual $\left(l_{1}, \xi\right)$ crack parameters using any two of these three frequencies.

In the majority of cases, the two methods cannot provide a unique value for the non-dimensional crack position $l_{1}^{*}$, which require usually an initial guess closest to the solution. This is due to the symmetric of crack locations 
(i.e., 0.2 and 0.8) when the boundary conditions are also symmetric (i.e., simply supported).

Table 4.b. Comparison of predicted and actual crack parameters for various crack location and depth by using $\omega_{c 1}-\omega_{c 3}$.

\begin{tabular}{|c|c|c|c|c|c|}
\hline \multirow{2}{*}{ Case } & \multirow{2}{*}{ Methods } & \multicolumn{4}{|c|}{$\omega_{c 1}-\omega_{c 3}$} \\
\cline { 3 - 6 } & & $l_{1}^{*}$ & Error $(\%)$ & $\xi^{*}$ & Error $(\%)$ \\
\hline \multirow{2}{*}{1} & Method1 & 0.1000 & 0.0881 & 0.1056 & 5.6217 \\
\cline { 2 - 6 } & Method2 & 0.1005 & 0.5714 & 0.1499 & 49.9553 \\
\hline \multirow{2}{*}{2} & Method1 & 0.2000 & 0.0012 & 0.3128 & 4.2958 \\
\cline { 2 - 6 } & Method2 & 0.2081 & 4.0728 & 0.4027 & 34.2600 \\
\hline \multirow{2}{*}{3} & Method1 & 0.2999 & $\approx 0$ & 0.2099 & 4.9609 \\
\cline { 2 - 6 } & Method2 & 0.3005 & 0.1801 & 0.2887 & 44.3610 \\
\hline \multirow{2}{*}{4} & Method1 & 0.2999 & 0.0001 & 0.3128 & 4.2944 \\
\cline { 2 - 6 } & Method2 & 0.3012 & 0.4000 & 0.4097 & 36.5950 \\
\hline \multirow{2}{*}{5} & Method1 & 0.3999 & 0.0002 & 0.2099 & 4.9624 \\
\cline { 2 - 6 } & Method2 & 0.3994 & 0.1495 & 0.2883 & 44.1763 \\
\hline \multirow{2}{*}{6} & Method1 & 0.4000 & $\approx 0$ & 0.4126 & 3.1732 \\
\cline { 2 - 6 } & Method2 & 0.3974 & 0.6369 & 0.5105 & 27.6268 \\
\hline \multirow{2}{*}{7} & Method1 & 0.2467 & 50.6406 & 0.2941 & 47.0685 \\
\cline { 2 - 6 } & Method2 & 0.4817 & 3.6403 & 0.2883 & 44.1548 \\
\hline \multirow{2}{*}{8} & Method1 & 0.2362 & 52.7556 & 0.5375 & 34.3815 \\
\cline { 2 - 6 } & Method2 & 0.4646 & 7.0734 & 0.5093 & 27.3352 \\
\hline
\end{tabular}

It is observed that the crack identification results for normalized crack location are relatively acceptable. The errors in the $7^{\text {th }}$ and 8 th cases are generally the highest for the $1^{\text {st }}$ method.

It is obvious that the crack identification results for normalized crack depth are not satisfactory; this is due to the deferred behavior of the flexibility coefficient.

Table 4.c. Comparison of predicted and actual crack parameters for various crack location and depth by using $\omega_{c 2}-\omega_{c 3}$.

\begin{tabular}{|c|c|c|c|c|c|}
\hline \multirow{2}{*}{ Case } & \multirow{2}{*}{ Methods } & \multicolumn{4}{|c|}{$\omega_{c 2}-\omega_{c 3}$} \\
\cline { 3 - 6 } & & $l_{1}^{*}$ & Error $(\%)$ & $\xi^{*}$ & Error $(\%)$ \\
\hline \multirow{2}{*}{1} & Method1 & 0.1002 & 0.2473 & 0.1054 & 5.4543 \\
\cline { 3 - 6 } & Method2 & 0.1005 & 0.5028 & 0.1500 & 50.0562 \\
\hline \multirow{2}{*}{2} & Method1 & 0.2000 & 0.0041 & 0.3128 & 4.2935 \\
\cline { 2 - 6 } & Method2 & 0.2097 & 4.8554 & 0.4007 & 33.5849 \\
\hline \multirow{2}{*}{3} & Method1 & 0.2999 & 0.0004 & 0.2099 & 4.9612 \\
\cline { 2 - 6 } & Method2 & 0.3031 & 1.0639 & 0.2871 & 43.5758 \\
\hline \multirow{2}{*}{4} & Method1 & 0.2999 & 0.0005 & 0.3128 & 4.2947 \\
\cline { 2 - 6 } & Method2 & 0.3070 & 2.3661 & 0.4056 & 35.2128 \\
\hline \multirow{2}{*}{5} & Method1 & 0.4000 & 0.0002 & 0.2099 & 4.9622 \\
\cline { 2 - 6 } & Method2 & 0.4006 & 0.1726 & 0.2880 & 44.0040 \\
\hline \multirow{2}{*}{6} & Method1 & 0.3999 & 0.0001 & 0.4126 & 3.1733 \\
\cline { 2 - 6 } & Method2 & 0.4028 & 0.7158 & 0.5087 & 27.1855 \\
\hline \multirow{2}{*}{7} & Method1 & 0.5003 & 0.0652 & 0.2099 & 4.9610 \\
\cline { 2 - 6 } & Method2 & 0.5 & 0 & 0.2878 & 43.9407 \\
\hline \multirow{2}{*}{8} & Method1 & 0.4998 & 0.0322 & 0.4115 & 2.8772 \\
\cline { 2 - 6 } & Method2 & 0.5 & 0 & 0.5073 & 26.8415 \\
\hline
\end{tabular}

\section{Conclusion}

Two methods used to solve the direct and inverse problems of a simply supported beam with one crack are compared in this paper. The Euler-Bernoulli beam theory is employed to model the cracked beam and the crack is represented as a rotational spring. This study allows making the following conclusions:

- The frequencies decreased when the crack is deeper;

- The crack depth has no effects on the second natural frequencies at the mid-span;

- The natural frequencies predicted by the two methods agree well with those obtained from previous work but the error increases for higher modes of vibration;

- The crack parameters predicted in the numerical examples show that both methods are theoretically capable of estimating the location of cracks and with less performance the crack depths.

\section{References}

1. A.D. Dimarogonas, Engineering Fracture Mechanics, 55:5 831-857 (1996)

2. R. Gasch, Journal of Sound and Vibration, 160(2) 313-332 (1993)

3. W. Ostachowicz, M. Krawczuk, Key Engineering Materials, 204-205 185-200 (2001)

4. M.I. Friswell, J.E.T. Penny Jet, Structural Health Monitoring, 1:2 139-148 (2002)

5. W.M. Ostachowicz, M. Krawczuk, Journal of Sound and Vibration, 150:2 191-201 (1991)

6. P.F. Rizos, N. Aspragathos, A.D. Dimarogonas, Journal of Sound and Vibration, 138:3 381-388 (1990)

7. T.G. Chondros, A.D. Dimarogonas, J. Yao, Journal of Sound and Vibration, 215:1 17-34 (1998)

8. O.S. Salawu, Engineering Structures, 19 718-723 (1997)

9. A.K. Pandey, M. Biswas, Journal of Sound and Vibration, 169(1) 3-17 (1994)

10. A.K. Pandey, M. Biswas, M.M. Samman, Journal of Sound and Vibration, 145(2) 321-332 (1991)

11. R.Y. Liang, F.K. Choy, J. Hu, Journal of the Franklin Institute, 328 505-518 (1991)

12. B.P. NANDWANA, S.K. MAITI, Journal of Sound and Vibration, 203:3 435-446 (1997)

13. J. Lee, Journal of Sound and Vibration, 320 482-490 (2009)

14. M. Attar, International Journal of Mechanical Sciences, 57 19-33 (2012)

15. J. Ferna Ndez-Sa'Ez, L. Rubio, C. Navarro, Journal of Sound and Vibration, 225 345-352 (1999)

16. H.P. Lin, Engineering Structures, 26 427-436 (2004)

17. J. Hu, R.Y. Liang, Journal of the Franklin Institute, 330(5) 841-853 (1993)

18. J. Xiang, Y. Zhong, X. Chen, Z. He, International Journal of Solids and Structures, 45 4782-4795 (2008)

19. J.W. Xiang, X.F. Chen, B. Li, YM. He, Z.J. He, Journal of Sound and Vibration, 296 1046-1052 (2006) 